MATEC Web of Conferences 1, 00001 (2012)

DOI: $10.1051 /$ matecconf/20120100001

(C) Owned by the authors, published by EDP Sciences, 2012

\title{
Crack's bifurcation in an anisotropic material
}

\author{
F.Lahna ${ }^{1}$, N.Belrhazi ${ }^{1}$, A. El Kaaba ${ }^{1}$ \\ ${ }^{1}$ F.S.A.C, Laboratoire de mécanique, Km 8 route d’El Jadida BP 5366 Mâarif, Casablanca, Maroc
}

\begin{abstract}
The orthotropic of a solid is characterized by the material directions $(\overrightarrow{1}, \overrightarrow{2}, \overrightarrow{3})$ and corresponds to six main directions of the crack' distribution 21, 31, 32, 12, 13 and 23 (the first number corresponds to the normal of the crack's plan, the second in the crack's front). In this work, it was suggested studying the crack's bifurcation solicited in the mixed mode of an anisotropic fracture's specimen by varying several geometrical parameters. The study showed that the critical angle of bifurcation depends on the crack's length, the specimen's height, the angle of load as well as on the chosen configuration.
\end{abstract}

Keywords: fracture, crack, bifurcation, mixed mode, stress intensity factor, orthotropic, criterion of Sih.

\section{Introduction}

If the major part of the studies of the mechanics of the fracture concerns a crack solicited in mode 1, the situations met in mechanical constructions are often more complex. In other words, it can happen frequently that the crack is solicited in the mixed mode $(1+2)$.

It is thus justifiable to wonder how are going to be modified the usual criteria of distribution in mode 1 .

In this work, we are interested in the influence of some geometrical parameters (crack's length, the specimen's height and the angle of load), so as the six main distribution's direction on the direction of distribution of a crack solicited in mixed mode. The criterion used to make this study is the criterion of the strain density energy of Sih.

\section{Criterion of the strain density energy of Sih}

The figure 1 defines a system of coordinates in the crack's front and the stress acting on the element of surface dA (the thickness equal 1).

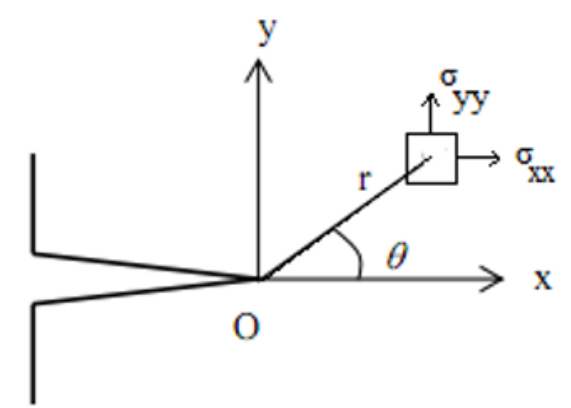

Fig.1. Applied stress on the bottom of a crack
One of the bases of this theory is that the fracture starts from a small element situated at the distance $r$ of the crack's front.

In the plan problem, for materials globally linear elastic, the strain density energy contained in the element $\mathrm{dA}$ is expressed by:

$\partial W / \partial A=1 / 2\left(\sigma_{11} \epsilon_{11}+\sigma_{22} \epsilon_{22}+2 \sigma_{12} \varepsilon_{12}\right)$

The $\sigma_{\mathrm{ij}}$ and $\varepsilon_{\mathrm{ij}}$ are respectively, the stresses and strains. The stresses in the crack's front express themselves according to the stress intensity factors $\mathrm{K}_{\mathrm{I}}$ and $\mathrm{K}_{\mathrm{II}}$ :

$\left\{\begin{array}{l}\sigma_{11}=\frac{K_{1}}{\sqrt{2 r}} A+\frac{K_{2}}{\sqrt{2 r}} B \\ \sigma_{22}=\frac{K_{1}}{\sqrt{2 r}} C+\frac{K_{2}}{\sqrt{2 r}} D \\ \sigma_{12}=\frac{K_{1}}{\sqrt{2 r}} E+\frac{K_{2}}{\sqrt{2 r}} F\end{array}\right.$
With:

$\left\{\begin{array}{l}A=\frac{1}{\sqrt{\pi}} \operatorname{Re}\left[\frac{\mu_{1} \mu_{2}}{\mu_{1}-\mu_{2}}\left(\frac{\mu_{2}}{z_{2}}-\frac{\mu_{1}}{z_{1}}\right)\right] \\ B=\frac{1}{\sqrt{\pi}} \operatorname{Re}\left[\frac{\mu_{1} \mu_{2}}{\mu_{1}-\mu_{2}}\left(\frac{\mu_{2}^{2}}{z_{2}}-\frac{\mu_{1}^{2}}{z_{1}}\right)\right] \\ C=\frac{1}{\sqrt{\pi}} \operatorname{Re}\left[\frac{1}{\mu_{1}-\mu_{2}}\left(\frac{\mu_{1}}{z_{2}}-\frac{\mu_{2}}{z_{1}}\right)\right] \\ D=\frac{1}{\sqrt{\pi}} \operatorname{Re}\left[\frac{1}{\mu_{1}-\mu_{2}}\left(\frac{1}{z_{2}}-\frac{1}{z_{1}}\right)\right] \\ E=\frac{1}{\sqrt{\pi}} \operatorname{Re}\left[\frac{\mu_{1} \mu_{2}}{\mu_{1}-\mu_{2}}\left(\frac{1}{z_{1}}-\frac{1}{z_{2}}\right)\right] \\ F=\frac{1}{\sqrt{\pi}} \operatorname{Re}\left[\frac{1}{\mu_{1}-\mu_{2}}\left(\frac{\mu_{1}}{z_{1}}-\frac{\mu_{2}}{z_{2}}\right)\right]\end{array}\right.$

Where the complex coefficients $\mu_{\mathrm{i}}$ are solutions of the characteristic equation:

$d_{11} \mu^{4}-2 d_{16} \mu^{3}+\left(2 d_{12}+d_{66}\right) \mu^{2}-2 d_{26} \mu+d_{22}=0$

$z_{k}=\left(\cos (\theta)+\mu_{k} \sin (\theta)\right)^{1 / 2} ; \quad k=1,2$ 
$R e$ is indicating the real part of [...].

The $d_{i j}$ are the coefficients of compliance such as in the plan $(\vec{\imath}, \vec{\jmath})$ we have:

$\left\{\begin{array}{l}\epsilon_{11} \\ \epsilon_{22} \\ \epsilon_{12}\end{array}\right\}=\left(\begin{array}{lll}d_{11} & d_{12} & d_{16} \\ d_{12} & d_{22} & d_{26} \\ d_{16} & d_{26} & d_{66}\end{array}\right)\left\{\begin{array}{l}\sigma_{11} \\ \sigma_{22} \\ \sigma_{12}\end{array}\right\}$

The strain density energy of Sih is $[1,2]$ :

$\mathrm{S}=\mathrm{r} \frac{\partial \mathrm{W}}{\partial \mathrm{A}}$

So:

$S=M_{11} K_{I}^{2}+M_{22} K_{I I}^{2}+2 M_{12} K_{I} K_{I I}$

With:

$M_{11}=\frac{1}{4}\left[d_{11} A^{2}+d_{22} C^{2}+d_{66} E^{2}+2 d_{12} A C+2 d_{16} A E+\right.$ $\left.2 d_{26} C E\right]$

$M_{22}=\frac{1}{4}\left[d_{11} B^{2}+d_{22} D^{2}+d_{66} F^{2}+2 d_{12} B D+2 d_{16} B F+\right.$ $\left.2 d_{26} D F\right] \quad(10)$

$M_{12}=\frac{1}{4}\left[d_{11} A B+d_{22} C D+d_{66} E F+d_{12}(A D+B C)+\right.$ $d_{16}(A F+B E)+d_{26}(C F+D E](11)$

According to the criterion of Sih, the direction of bifurcation $\theta_{c}$ is obtained when $S$ reaches a stationary value:

$\frac{\partial S}{\partial \theta}=0$ en $\theta=\theta_{c}$

\section{Application of the criterion of Sih}

The application of this criterion requires the knowledge of the modulus of elasticity of the material tested as well as the expressions of the stress intensity factors $\mathrm{K}_{\mathrm{I}}$ and $\mathrm{K}_{\mathrm{II}}$ of the studied specimen.

\subsection{Studied material}

The orthotropic material tested in this study is defined by the following mechanical characteristics [3]:

$$
\begin{aligned}
& E_{1}=11370[\mathrm{MPA}], E_{2}=944[\mathrm{MPA}], E_{3}=703[\mathrm{MPA}], \\
& G_{12}=1350[\mathrm{MPA}], G_{13}=1615[\mathrm{MPA}], G_{23}=200[\mathrm{MPA}], \\
& v_{12}=0.32, v_{13}=0.53, v_{23}=0.58,
\end{aligned}
$$

\subsection{Specimen used}

To lead this study, the choice was made on a specimen, very used in fracture's mechanics; it is about the specimen DCB (double cantilever beam). The figure 2 defines the dimensions, the geometrical base of the specimen as well as the angle of load $\beta\left(\beta=90^{\circ}\right.$ if it's mode $1,0^{\circ}<\beta<90^{\circ}$ if it's mixed mode $1+2$ and $\beta=0^{\circ}$ if it's mode 2):

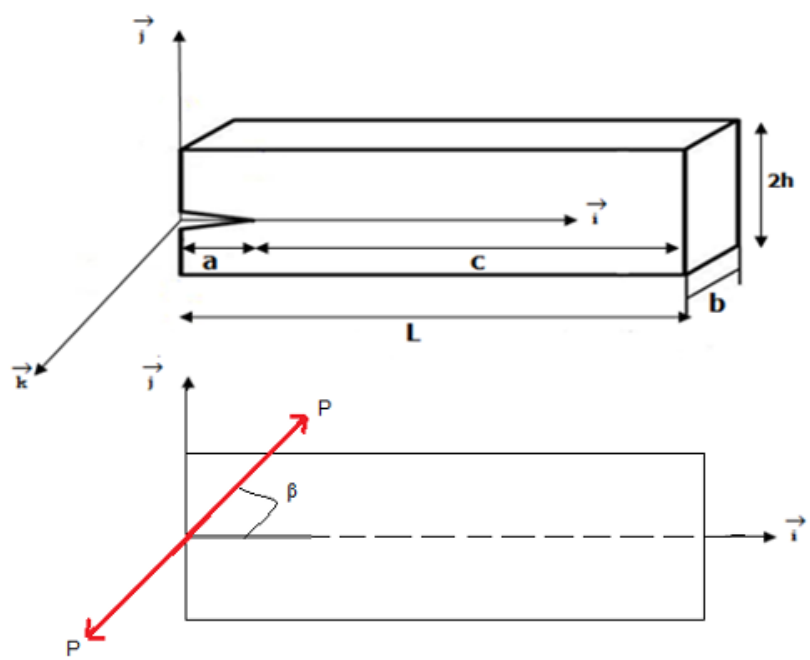

Fig.2. specimen double cantilever beam

- a : Length of the crack (mm)

- L: Length of the specimen (mm)

- 2h:Specimen's height (mm)

- P: Applied load (daN)

- $\quad \beta$ : Angle of load $\left(^{\circ}\right)$

\subsection{The stress intensity factor}

The analytical expression of the FIC $[4,5,6]$ (for the mode I and mode II) is given by equation (16) and (17):

$$
\mathrm{K}_{\mathrm{I}}=\frac{P \sqrt{\frac{12}{(\lambda c)^{3}}\left[\left(\frac{\lambda a\left(\operatorname{sh}^{2}(\lambda c)+\sin ^{2}(\lambda c)\right)+\operatorname{sh}(\lambda c) \operatorname{ch}(\lambda c)-\sin (\lambda c) \cos (\lambda c)}{\operatorname{sh}^{2}(\lambda c)-\sin ^{2}(\lambda c)}\right)\right]^{2}+\frac{3 r}{h}}}{b\left(\frac{\alpha_{22}}{2 \alpha_{11}}\right)\left[\sqrt{\frac{\alpha_{22}}{\alpha_{11}}}+\frac{2 \alpha_{12}+\alpha_{66}}{2 \alpha_{11}}\right]^{\frac{1}{4}}}
$$$$
K_{I I}=\left(\frac{4 P}{h b^{2}}\right)^{\frac{1}{2}} \cdot\left\{\frac{1}{2}\left[\left(\frac{\alpha_{22}}{\alpha_{11}}\right)^{\frac{1}{2}}+\frac{2 \alpha_{12}+\alpha_{66}}{2 \alpha_{11}}\right]^{\frac{1}{2}}\right\}
$$

\section{Results and conclusions}

We give below the results obtained for six configurations of orthotropic for various crack's length a $(60 \leq \mathrm{a} \leq 220)$, for different the specimen's height $h(40 \leq 2 \mathrm{~h} \leq 100)$ and for different angle of load $\beta\left(0^{\circ} \leq \beta \leq 90^{\circ}\right)$.

\subsection{Variation of $S$ according to $\theta$}

The study of the curves $S=f(\theta)$ shows that for 3 modes of load we have the following situations:

a- For the mode $2\left(\beta=0^{\circ}\right)$, the curves $S(\theta)$ obtained for the various values of "a" and of " $h$ "; and for the six considered configurations have the same form as that of the figure 3 . It is a symmetric curve characterized by two opposite minimums 
$\left( \pm \theta_{c}\right)$ which define two possible angles of bifurcation (figure3).

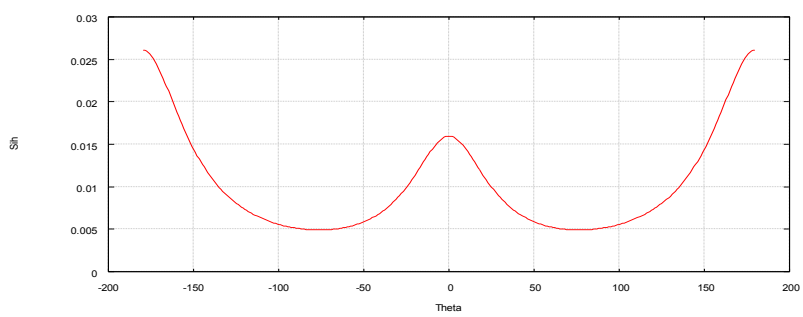

Fig.3.Case of the mode 2

b- For the modes 1 and $1+2\left(0^{\circ}<\beta<90^{\circ}\right)$. The curves $S(\theta)$ obtained for the various values of ' $a$ ' and of $h$ have the same form and present a maximum for the configurations 21 and 31 (figure 4), and a minimum for the configurations 32, 12, 13 and 23 (figure 5).

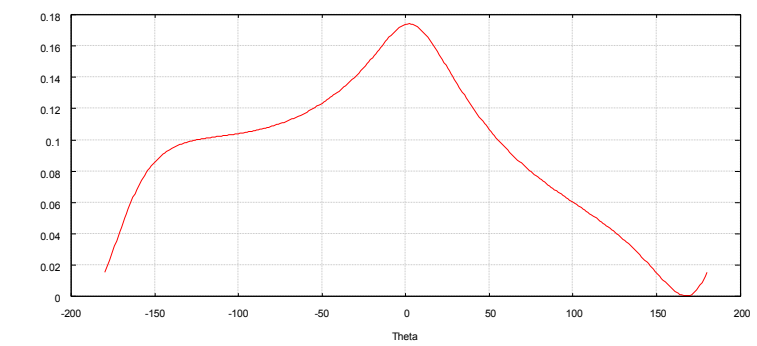

Fig.4.Case of the mixed mode $(21,31)$

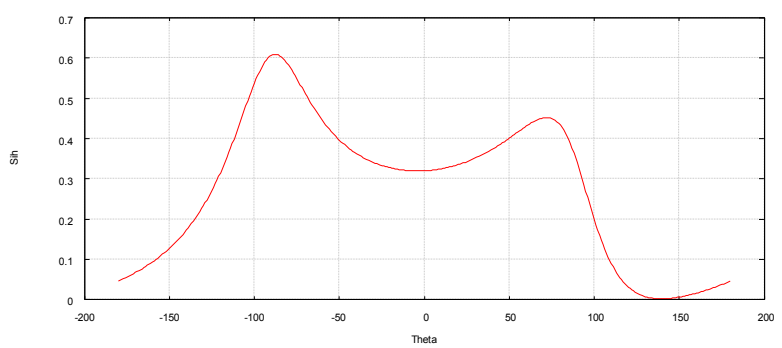

Fig.5.Case of the mixed mode (32, 12, 13 and 23)

\subsection{Influence of the crack's length ' $a$ '}

The study of the results obtained for six configurations, allows us to make the following observations:

For the modes 1 and 2, the bifurcation's angle $\theta_{\mathrm{c}}$ are independent from the crack's length ' $a$ '. In mode $1 \theta_{\mathrm{c}}=0^{\circ}$ and in mode $2 \theta_{\mathrm{c}} \approx \pm 80^{\circ}$.

- For the mixed mode $\left(0^{\circ}<\beta<90^{\circ}\right)$, the bifurcation's angle $\theta_{\mathrm{c}}$ decreases when the crack's length increases. The figure 6 gives the curves $\theta_{c}=\theta_{c}$ (a) for the six considered configurations. These curves show that if for the four configurations $21,31,13$ and 12 we have a low variation of $\theta_{c}$; it is not there so for the two other configurations $(32,23)$.

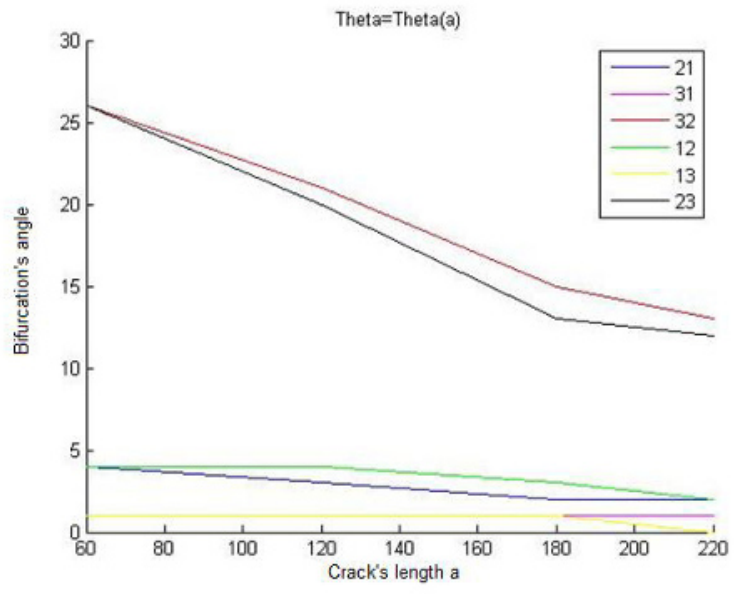

Fig.6. $\theta_{\mathrm{c}}=\theta_{\mathrm{c}}(\mathrm{a})$

\subsection{Influence of the specimen's height $h$}

The study of the results obtained for the 6 configurations, allows us to make the following observations:

- For the modes 1 and 2, the bifurcation's angles $\theta_{\mathrm{c}}$ are independent of $\mathrm{h}$. In mode $1 \theta_{\mathrm{c}}=0^{\circ}$ and in mode $2 \theta_{\mathrm{c}} \approx \pm 80^{\circ}$.

- For the mixed mode $\left(0^{\circ}<\beta<90^{\circ}\right)$, the angle of bifurcation $\theta_{\mathrm{c}}$ increases when the specimen's height increases. The figure 7 gives the curves $\theta_{c}=\theta_{c}(a)$ for 6 considered configurations.

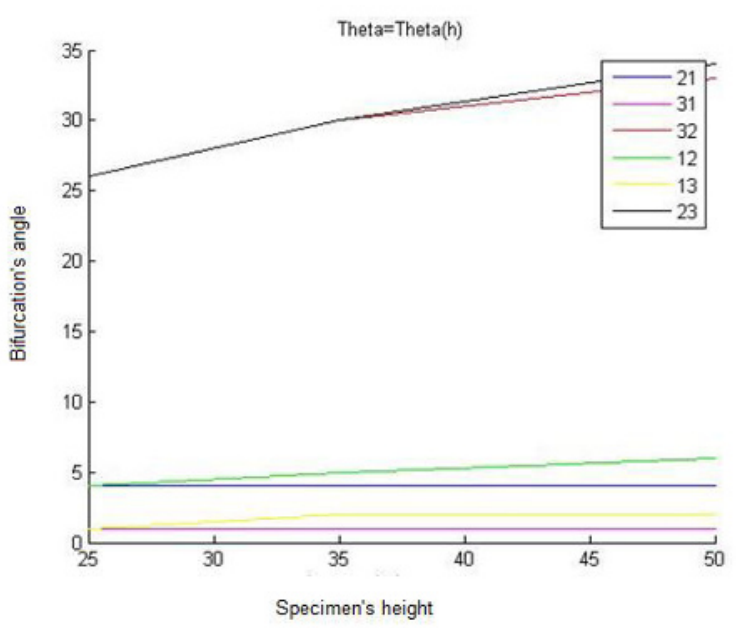

Fig.7. $\theta_{\mathrm{c}}=\theta_{\mathrm{c}}(\mathrm{h})$

\subsection{Influence of the angle of load $\beta$}

The study of the results obtained for six configurations allows us to make the following observations:

- For the modes 1 and 2, the bifurcation's angles $\theta_{\mathrm{c}}$ are independent from the angle of load $\beta$. In mode $1 \theta_{\mathrm{c}}=0^{\circ}$ and in mode $2 \theta_{\mathrm{c}} \approx \pm 75^{\circ}$.

- For the mixed mode $\left(0^{\circ}<\beta<90^{\circ}\right)$, the bifurcation's angle $\theta_{c}$ decreases when the angle of load $\beta$ increases. The figure 8 gives the curves $\theta_{\mathrm{c}}=\theta_{\mathrm{c}}(\beta)$ for 6 considered configurations. 


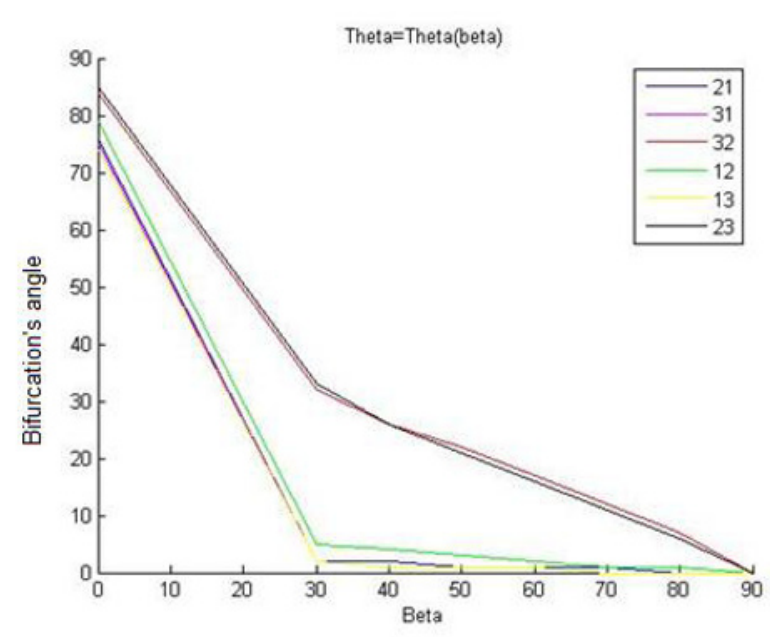

Fig.8. $\theta_{c}=\theta_{c}(\beta)$ orthotrope. CR Acad.Sci.Paris Série II 299(3) (1984) $85-$ 88.

6 F.LAHNA : Influence des orientations des axes d'orthotropie sur les facteurs d'intensité de contraintesapplication au bois. Congrès AUM Toulouse septembre 1999.

\section{Conclusion}

The application of the criterion of Sih in an anisotropic specimen DCB allowed us to make the following conclusions:

- Contrary to the isotropic materials, the bifurcation's angle $\theta_{\mathrm{c}}$ of a crack in an anisotropic material does not correspond always to a minimum of the curve $S(\theta)$ like on the configurations 21 and 31 .

- For the modes 1 and 2, the bifurcation's angle $\theta_{c}$ does not depend on the crack's length and the specimen's height.

- $\quad$ For the mixed mode 1+2, the bifurcation's angle $\theta_{c}$ decreases when the crack's length grows, and varies in the same sense as the specimen's height.

- For the six configurations tested, the bifurcation's angle decreases when the angle of load increases.

\section{References}

1 G.C. Sih : Strain energy density factor applied to mixed crack problems- International Journal of fracture, vol.10, No 3, September 1974,p.305-321.

2 G.C. Sih, E.P. Chen, Fracture analysis of unidirectional composites, J. of Composite Materials, Vol. 7, pp 230244, april 1973.

3 G.Valentin, G. Adjanohoun: Applicability of classical isotropic fracture mechanics specimens to wod crack propagation studies -Materials and structures 1992, 25,313.

4 F.LAHNA : Mécanique de la rupture des matériaux orthotropes-application au bois. Thèse,Bordeaux, $\mathrm{n}^{\circ} 1876,1983$.

5 G.VALENTIN et F.LAHNA :Facteur d'intensité de contrainte de l'éprouvette DCB dans le cas d'un matériau 